\title{
Adipose triacylglycerol lipase is a major regulator of hepatic lipid metabolism but not insulin sensitivity in mice
}

\author{
S. M. Turpin • A. J. Hoy • R. D. Brown • C. Garcia Rudaz • \\ J. Honeyman • M. Matzaris $\cdot$ M. J. Watt
}

Received: 29 March 2010 /Accepted: 4 August 2010/Published online: 15 September 2010

(C) Springer-Verlag 2010

\begin{abstract}
Aims/hypothesis Hepatic steatosis is characterised by excessive triacylglycerol accumulation and is strongly associated with insulin resistance. An inability to efficiently mobilise liver triacylglycerol may be a key event mediating hepatic steatosis. Adipose triacylglycerol lipase (ATGL) is a key triacylglycerol lipase in the liver and we hypothesised that liver-specific overproduction of ATGL would reduce steatosis and enhance insulin action in obese rodents.

Methods Studies of fatty acid metabolism were conducted in primary hepatocytes isolated from wild-type and Atgl (also known as Pnpla2) ${ }^{-/-}$mice. An ATGL adenovirus was utilised to overproduce ATGL in the livers of obese insulinresistant C57Bl/6 mice (Ad-ATGL). Blood chemistry, hepatic lipid content and insulin sensitivity were assessed in mice. Results Triacylglycerol content was increased in $\mathrm{Atg}^{/-}$ hepatocytes and was associated with increased fatty acid uptake and impaired fatty acid oxidation. ATGL adenovirus administration in obese mice increased the production of hepatic ATGL protein and reduced triacylglycerol, diacylglycerol and ceramide content in the liver. Overproduction of ATGL improved insulin signal transduction in the liver but did
\end{abstract}

Electronic supplementary material The online version of this article (doi:10.1007/s00125-010-1895-5) contains supplementary material, which is available to authorized users.

S. M. Turpin · A. J. Hoy • R. D. Brown · C. Garcia Rudaz •

M. Matzaris $\cdot$ M. J. Watt $(\bowtie)$

Biology of Lipid Metabolism Laboratory,

Department of Physiology, Monash University,

Wellington Rd,

Clayton, Victoria 3800, Australia

e-mail: matthew.watt@med.monash.edu.au

S. M. Turpin · J. Honeyman

St Vincent's Institute of Medical Research

and the Department of Medicine, University of Melbourne,

Fitzroy, Victoria, Australia not affect fasting glycaemia or insulinaemia. Inflammatory signalling was not suppressed by ATGL overproduction. While ATGL overproduction increased plasma non-esterified fatty acids, neither lipid deposition nor insulin-stimulated glucose uptake were affected in skeletal muscle.

Conclusions/interpretation Liver ATGL overproduction decreases hepatic steatosis and mildly enhances liver insulin sensitivity. These effects are not sufficient to improve fasting glycaemia or insulinaemia in rodent obesity.

Keywords Fatty acid metabolism - Insulin resistance .

Obesity $\cdot$ Steatosis $\cdot$ Triacylglycerol

$\begin{array}{ll}\text { Abbreviations } \\ \text { ATGL } & \text { Adipose triacylglycerol lipase } \\ \text { ALT } & \text { Alanine aminotransferase } \\ \text { CGI } & \text { Comparative gene identification } \\ \text { JNK } & \text { c-Jun N-terminal kinase } \\ \text { FOXO } & \text { Forkhead box O } \\ \text { G6Pase } & \text { Glucose-6-phosphatase } \\ \text { HGP } & \text { Hepatic glucose production } \\ \text { HSL } & \text { Hormone sensitive lipase } \\ \text { NAFLD } & \text { Non-alcoholic fatty liver disease } \\ \text { PEPCK } & \text { Phosphoenolpyruvate kinase } \\ \text { PKC } & \text { Protein kinase C } \\ \text { SREBP } & \text { Sterol regulatory element binding protein } \\ \text { TAG } & \text { Triacylglycerol } \\ \text { TGH } & \text { Triacylglycerol hydrolase } \\ \text { Wt } & \text { Wild type }\end{array}$

\section{Introduction}

Obesity is accompanied by the accumulation of triacylglycerol (TAG) and other lipids in non-adipose tissues, including the skeletal muscle, pancreas and liver $[1,2]$. 
Hepatic steatosis is the accumulation of TAG in the liver and is a major element of non-alcoholic fatty liver disease (NAFLD). NAFLD affects $\sim 20 \%$ of the adult population and is found in over two-thirds of obese people [3]. Hepatic steatosis is strongly associated with insulin resistance, which is characterised by an inability of insulin to suppress hepatic glucose production (HGP) and stimulate glucose uptake into peripheral tissues such as skeletal muscle [4]. Elevated HGP due to the defective suppression of gluconeogenesis and impaired glucose uptake are primary defects contributing to fasting hyperglycaemia in type 2 diabetes $[5,6]$. The majority of studies in humans and animals have shown that insulin-resistant states can be accompanied by hepatic steatosis. For example, rodents fed a high-fat diet, $o b / o b$ mice and lipodystrophic mice all develop hepatic steatosis and insulin resistance [7-9]. However, recent data suggest that hepatic TAG accumulation per se is not sufficient to cause insulin resistance [10-12]; instead, it is the inflammation that often accompanies hepatic steatosis that is likely to be the actual cause of the insulin resistance.

The liver plays a key role in systemic lipid homeostasis through its ability to synthesise and store fatty acids as TAG, and to secrete TAG in the form of VLDL. Hepatic fatty acids are derived from several sources, including adipose tissue lipolysis, chylomicron-TAG lipolysis and de novo lipogenesis, and can be stored as TAG in lipid droplets located within the cytosol [13]. Hepatic lipases mobilise the fatty acids from these TAGs for oxidation, or for re-esterification to TAG at the endoplasmic reticulum where the TAG is packaged into an apolipoprotein-Bcontaining VLDL precursor for secretion [13-15]. The regulation of these key processes of hepatic TAG homeostasis remains poorly defined.

The metabolic events leading to the development of hepatic steatosis are unresolved. While decreases in fatty acid oxidation [16] or increases in fatty acid uptake [17] play important roles, another possibility is that TAG accumulates as a consequence of an inability to mobilise the stored TAG in the presence of increased fatty acid import and biosynthesis [14]. The first step in the mobilisation of TAG is catalysed by several lipases, which include adipose triacylglycerol lipase (ATGL), hormone sensitive lipase (HSL) and triacylglycerol hydrolase (TGH). ATGL is an essential TAG lipase in many cell types [18] and Atgl (also known as Pnpla 2$)^{-/-}$mice store vast quantities of TAG in the major metabolic tissues, including the liver [19]. Post-translational regulation of ATGL TAG lipase activity is mediated by ATGL association with comparative gene identification (CGI)-58 [20], which enhances ATGL activity and its association with lipid droplets [21]. While hepatic ATGL content is low, it is proposed to account for a third of TAG lipase activity [22] and Atgl overexpression ( $>200$-fold) in a hepatoma cell line or the livers of $o b / o b$ mice reduces TAG levels [22]. Similarly, CGI-58 enhances the use of stored TAG in hepatoma cells by facilitating the packaging of TAG into lipoprotein particles [23, 24].

Some clinical observations suggest a regulatory role of ATGL TAG lipase activity in steatosis and insulin resistance. ATGL and CGI-58 protein abundance is reduced in the livers of insulin-resistant patients with NAFLD [25] and genetic variations within the $A T G L$ gene are associated with type 2 diabetes and plasma TAG [26]. Together, these findings suggest that enhancing ATGL-mediated lipolysis in the liver may be a novel treatment for NAFLD and hepatic insulin resistance. We tested the hypothesis that ATGL is an important control point for TAG mobilisation in hepatocytes, and that overproduction of ATGL would reduce steatosis and enhance insulin action in the setting of obesity.

\section{Methods}

Animals Male C57B1/6 and ob/ob mice aged 10 weeks were purchased from Monash Animal Services (Clayton, VIC, Australia). $\mathrm{Atg} \mathrm{I}^{-1}$ mice were generated by targeted homologous recombination as described by Haemmerle et al. [19]. Mice were maintained on a $12 \mathrm{~h}$ light/dark cycle and had free access to regular chow and water. Male C57Bl/6 mice were fed a high-fat diet (59\% energy from fat; Specialty Feeds, Glen Forrest, WA, Australia) for 2428 weeks prior to experiments. Body composition was determined by dual-energy X-ray absorptiometry (Lunar PIXImus2 mouse densitometer; GE Healthcare, Piscataway, NJ, USA). The Monash University School of Biomedical Sciences Animal Ethics Committee approved all procedures and the principles of laboratory animal care were followed.

Primary hepatocyte isolation $A \operatorname{tg} \Gamma^{1-}$ mice $(n=6)$ and sexmatched wild-type (Wt) littermates $(n=6)$ aged 6-8 weeks were killed and hepatocytes isolated [27]. Hepatocytes were maintained in Medium 199 (Gibco, Invitrogen, Mulgrave, VIC, Australia) containing $20 \mathrm{ng} / \mathrm{ml}$ dexamethasone, $10 \mathrm{ng} / \mathrm{ml}$ epidermal growth factor, $10 \%$ (vol./vol.) fetal bovine serum and $1 \%$ glutamine and penicillin. Experiments were performed within 3 days of isolation.

Assessment of hepatocyte fatty acid metabolism and insulin sensitivity Hepatocytes were incubated for $6 \mathrm{~h}$ with Medium 199 containing 2\% fatty-acid-free bovine serum albumin, $\left[1-{ }^{14} \mathrm{C}\right]$ oleic acid $(18.5 \mathrm{MBq} / \mathrm{ml}$; GE Healthcare, Piscataway, NJ, USA) and $0.5 \mathrm{mmol} / \mathrm{l}$ unlabelled oleate to determine exogenous fatty acid oxidation. To determine endogenous fatty acid oxidation, hepatocytes were 'pulsed' for $24 \mathrm{~h}$ with the fatty acid medium described above to load endogenous lipid pools with $\left[{ }^{14} \mathrm{C}\right]$ oleate. The hepatocytes were washed with PBS then incubated for $6 \mathrm{~h}$ in fresh 
unlabelled fatty acid medium. Fatty acid oxidation was determined by measuring ${ }^{14} \mathrm{CO}_{2}$ in the culture media and acid soluble metabolite production. Fatty acid incorporation into TAG was assessed by a Folch extraction of cellular lipids followed by thin layer chromatography [28]. Fatty acid uptake was calculated as the sum of $\left[{ }^{14} \mathrm{C}\right]$ oleate oxidation and ${ }^{14} \mathrm{C}$ incorporation into all lipids. Hepatic insulin sensitivity was assessed by measuring hepatic glucose output and the suppression of Pepck and G6Pase (also known as $G 6 p c$ ) mRNA expression with insulin. Hepatocytes were incubated without or with increasing concentrations of insulin for $4 \mathrm{~h}$ before lysing in Qiazol lysis reagent (Qiagen, Doncaster, VIC, Australia).

Adenoviral vectors and administration The recombinant adenoviral vectors were Ad-ATGL expressing wild-type $\operatorname{Atgl}\left(1.7 \times 10^{11} \mathrm{pfu} / \mathrm{ml}\right)$ and Ad-GFP $\left(2.9 \times 10^{11} \mathrm{pfu} / \mathrm{ml}\right)$. See Electronic supplementary material (ESM) for cloning and adenovirus production. Adenovirus was injected into the jugular vein of anaesthetised mice $(2.2 \%$ [vol./vol.] isoflurane in $\mathrm{O}_{2}$ ) at a final concentration of $1.5 \times 10^{9} \mathrm{pfu} / \mathrm{g}$.

Insulin and pyruvate tolerance tests Insulin tolerance tests were conducted 12 days after adenovirus administration. Mice were fasted for $4 \mathrm{~h}$ and insulin $(1.0 \mathrm{U} / \mathrm{kg}$ body mass, Actrapid; Novo Nordisk, Baulkham Hills NSW, Australia) or pyruvate $(0.35$ or $2 \mathrm{mg} / \mathrm{kg})$ were injected into the intraperitoneal cavity. Blood glucose levels were measured from a tail cut (Accu-Chek; Roche, Mannheim, Germany).

Glucose uptake in isolated skeletal muscle Mice were fasted for $4 \mathrm{~h}$ and anaesthetised with an i.p. injection $(60 \mathrm{mg} / \mathrm{kg})$ of pentobarbital sodium. The soleus muscles were excised and glucose uptake was assessed for $10 \mathrm{~min}$ using 2-deoxy-D-[2,6- $\left.{ }^{3} \mathrm{H}\right]$ glucose $(1 \mathrm{mmol} / \mathrm{l}, 18.5 \mathrm{MBq} / \mathrm{ml})$ in the presence or absence of $10 \mathrm{nmol} / \mathrm{l}$ insulin.

Plasma biochemistry Plasma insulin was determined using a rat/mouse insulin ELISA (Millipore, Billerica, MA, USA). Plasma NEFA (Wako Chemicals, Wako, VA, USA) and TAG (Triacylglycerol E kit; Wako Chemicals) were determined by enzymatic colorimetric assays. Fasting plasma levels of alanine aminotransferase (ALT) was determined using ALT assay kits (Thermo Electron, Melbourne, VIC, Australia). TAG secretion was performed as described by Reid et al. [22].

Quantitative real-time PCR RNA was extracted from whole liver or hepatocytes using Qiazol and $1 \mu \mathrm{g}$ mRNA was reverse transcribed (iScript cDNA Synthesis Kit, BioRad Laboratories, Hercules, CA, USA). Quantitative real-time PCR was performed on a realplex Mastercycler (Eppendorf, North Ryde, NSW, Australia) using the TaqMan Universal
PCR Master Mix and TaqMan Gene Expression Assays (Applied Biosystems, Foster City, CA, USA; ESM Table 1). The relative quantification was calculated using the $\Delta \Delta \mathrm{C}_{\mathrm{t}}$ method, normalising values to Ad-GFP (in vivo studies) or Wt (in vitro studies).

Western blotting Tissues were rapidly dissected and frozen in liquid $\mathrm{N}_{2}$. Tissues were homogenised in ice cold RIPA lysis buffer, centrifuged and the supernatant fraction was resolved by SDS-PAGE and immunoblotted. See the ESM for detailed methods.

Tissue lipid analysis Livers were sectioned and stained for Oil Red O. For TAG quantification, lipids were extracted by a Folch extraction, the lipid reconstituted in $100 \%$ ethanol and TAG content determined using the TG-GPOPAP reagent (Roche). Diacylglycerol and ceramide content was determined by an $\left[{ }^{32} \mathrm{P}\right]$ ATP-linked enzymatic method as described by Watt et al. [29].

Statistics Values are expressed as means \pm SEM. Differences were tested by unpaired two-tailed $t$ tests or two-way analysis of variance with Bonferroni post hoc analysis (GraphPad Prism version 5.02). Statistical significance was set a priori at $p<0.05$.

\section{Results}

ATGL is decreased in the livers of obese mice Liver TAG was elevated in high-fat-fed and $o b / o b$ mice (Fig. 1a). High-fat-fed mice exhibited a marked reduction in liver Atgl (Fig. 1b), but there were no changes in the expression of other important TAG lipases, $\mathrm{Hsl}$ (also known as Lipe; Fig. 1c) or Tgh (also known as Ces3; Fig. 1d). Similar expression patterns were noted in the livers of $o b / o b$ mice compared with lean littermates (Fig. 1e-g). TAG content was increased in the liver of $\mathrm{Atg} \mathrm{I}^{-1-}$ mice, indicative of an important role for ATGL in modulating liver TAG content (Fig. 1h).

Atg $\Gamma^{/-}$hepatocytes exhibit defects in fatty acid metabolism and are steatotic Isolated hepatocytes from $\mathrm{Atg} \mathrm{I}^{--}$mice exhibited marked TAG accumulation (Fig. 2a). Diacylglycerol was increased $(1.00 \pm 0.05$ vs $1.97 \pm 0.12$ arbitrary units, $p<0.05)$ and ceramide decreased $(1.00 \pm 0.04$ vs $0.77 \pm 0.06$ arbitrary units, $p<0.05)$ in $A t g I^{/-}$hepatocytes. Fatty acid uptake was increased in $\mathrm{Atgl}^{-1-}$ hepatocytes (Fig. 2b) but fatty acid oxidation was decreased (Fig. 2c). The reduction in fatty acid oxidation was not due to defects in the contents of key mitochondrial enzymes or regulators of mitochondrial transcription, which were, surprisingly, increased (Fig. 2d). Activating phosphorylation of 5'-AMP-activated 

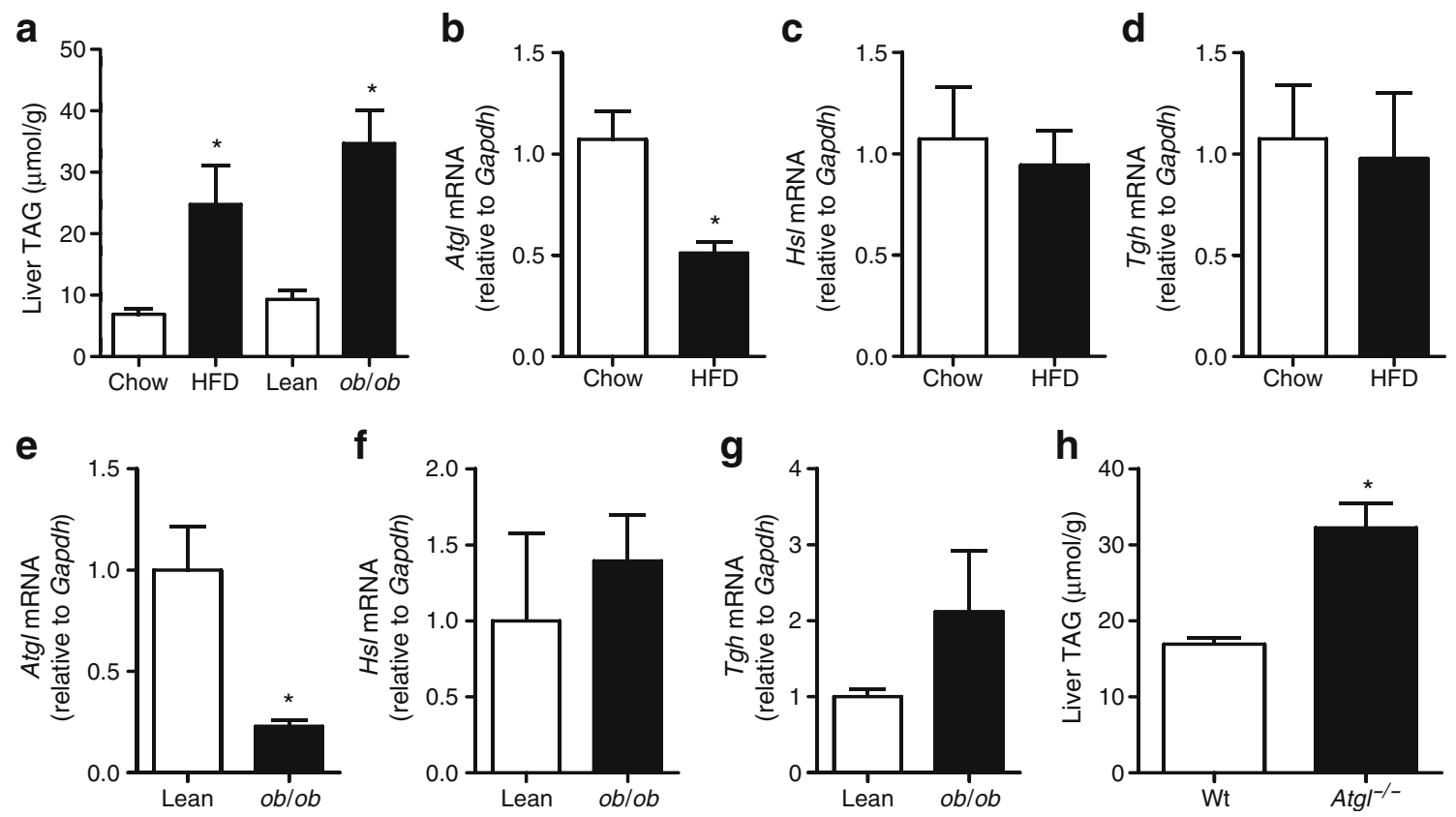

Fig. 1 ATGL is dysregulated in obese insulin-resistant rodents. a Liver triacylglycerol content in $\mathrm{C} 57 \mathrm{Bl} / 6$ mice fed a chow or high-fat diet (HFD) and $o b / o b$ mice. ${ }^{*} p<0.05, n=4$ per group. (b) Atgl, (c) Hsl and (d) Tgh mRNA expression in lean mice fed a chow diet and agematched littermates made obese by feeding a high-fat diet. ${ }^{*} p<0.05$,

$n=4-10$ per group. (e) $\mathrm{Atgl}$, (f) $\mathrm{Hsl}$ and (g) $\mathrm{Tgh}$ mRNA expression in obese $o b / o b$ mice and lean littermates. ${ }^{*} p<0.05, n=5$ per group. h Liver triacylglycerol content in $\operatorname{Atg}^{/-}$mice and wild-type agematched littermates. ${ }^{*} p<0.05, n=3$ per group

protein kinase on T172 of the $\alpha$-subunit was not different (Fig. 2e). Instead, the decreases in fatty acid oxidation were associated with increased fatty acid incorporation into TAG (Fig. 2f). This was not explained by increased levels of transcripts for proteins involved in fatty acid uptake, storage or TAG lipolysis (Fig. 2g). We investigated whether fatty acids stored within TAG could be efficiently catabolised in the absence of ATGL. TAG-derived fatty acid oxidation was reduced by $85 \%$ in $A \operatorname{tg} \Gamma^{/-}$hepatocytes (Fig. 2h). TAG secretion was not affected by Atgl ablation (Fig. 2i). This finding indicates that ATGL is a rate-limiting TAG lipase in hepatocytes that is important for providing substrate for fatty acid oxidation, but not TAG synthesis or export [22].

Atg ${ }^{-/}$hepatocytes are not insulin resistant Despite storing vast quantities of TAG in most tissues, whole-body $\mathrm{Atg} \mathrm{I}^{--}$ mice were originally reported to be insulin sensitive in multiple tissues including the liver [19]; however, more recent studies indicate that the liver of $\operatorname{Atg} \Gamma^{1-}$ mice may in fact be insulin resistant [30]. We examined insulin sensitivity in hepatocytes isolated from $\mathrm{Atg} \Gamma^{1-}$ and Wt mice, which precludes interference from systemic factors and local inflammatory mediators (e.g. Kupffer cells and mast cells). Insulin-stimulated phosphorylation of Akt at S473 and T308 was not different between genotypes (Fig. 3a, b). Consistent with this notion, the capacity of insulin to suppress phosphoenolpyruvate kinase (Pepck) and glucose-6-

phosphatase (G6Pase) mRNA expression was not different (Fig. 3c, d). HGP was lower in $A t g \Gamma^{/-}$hepatocytes (main effect, $p<0.05$ ) and was decreased by insulin in both genotypes (Fig. 3e). Suppression of HGP by insulin was not different between genotypes (Fig. 3f). Insulin-stimulated Akt phosphorylation was not affected in mice injected with insulin in vivo (ESM Table 2). Together, these findings indicate that despite inducing marked steatosis, Atgl ablation does not influence insulin sensitivity in hepatocytes.

ATGL overproduction in the livers of mice alters hepatic lipid metabolism We next tested the hypothesis that increasing ATGL production in the liver modulates lipid and glucose metabolism in vivo. Adenoviral ATGL overproduction was restricted to the liver, where it increased Atgl mRNA (Fig. 4a) and ATGL protein content (Fig. 4b). $H s l$ mRNA expression was decreased $(31 \%, p=0.02)$ and $T g h$ mRNA tended to increase $(50 \%, p=0.10)$ with AdATGL (data not shown). Adenoviral overexpression of Atgl in the liver did not affect food intake (Table 1). Similarly, body mass and fat mass in Ad-ATGL (Ad-GFP, 33.7 $\pm 1.2 \%$ body mass; Ad-ATGL, $32.7 \pm 1.8 \%, n=4, p=0.69$ ) were not different from mice treated with Ad-GFP (Table 1). Serum ALT levels were not different between groups (Table 1).

Overproduction of ATGL reduced liver steatosis in obese mice as denoted by less Oil Red O staining of neutral lipids (Fig. 4c). The lipid droplets containing TAG appeared 
Fig. 2 Atgl deletion alters hepatic lipid metabolism in vitro. Hepatocytes isolated from $\mathrm{Wt}$ and $\operatorname{Atg} \Gamma^{\prime-}$ mice were cultured for 2-3 days prior to functional analysis of fatty acid metabolism using radiolabelled oleate. a Hepatocyte triacylglycerol content ( $n=6$ per group).

(b) Uptake and (c) oxidation of fatty acids ( $n=8-12$ per group). d The mRNA of oxidative metabolism and mitochondrial biogenesis proteins was quantified by qRT-PCR and normalised against Gapdh ( $n=6$ per group). Gapdh was not different between groups (Wt: $20.3 \pm 0.4$ vs $\operatorname{Atg} \Gamma^{/-}: 19.6 \pm 0.6 \mathrm{AU}$, $p=0.34$ ). e 5'-AMP protein kinase T172 phosphorylation ( $n=3-4$ per group). f Fatty acid incorporation of media-derived fatty acids into intracellular triacylglycerol $(n=12$ per group). g The mRNA content of fatty acid synthesis/storage/ triacylglycerol lipase proteins ( $n=4-5$ per group). h Oxidation of fatty acid derived from intracellular stores (presumably TAG, $n=12$ per group). i TAG secretion into culture media over $24 \mathrm{~h}$ ( $n=4-6$ per group).

$*$ Different from Wt, $p<0.05$
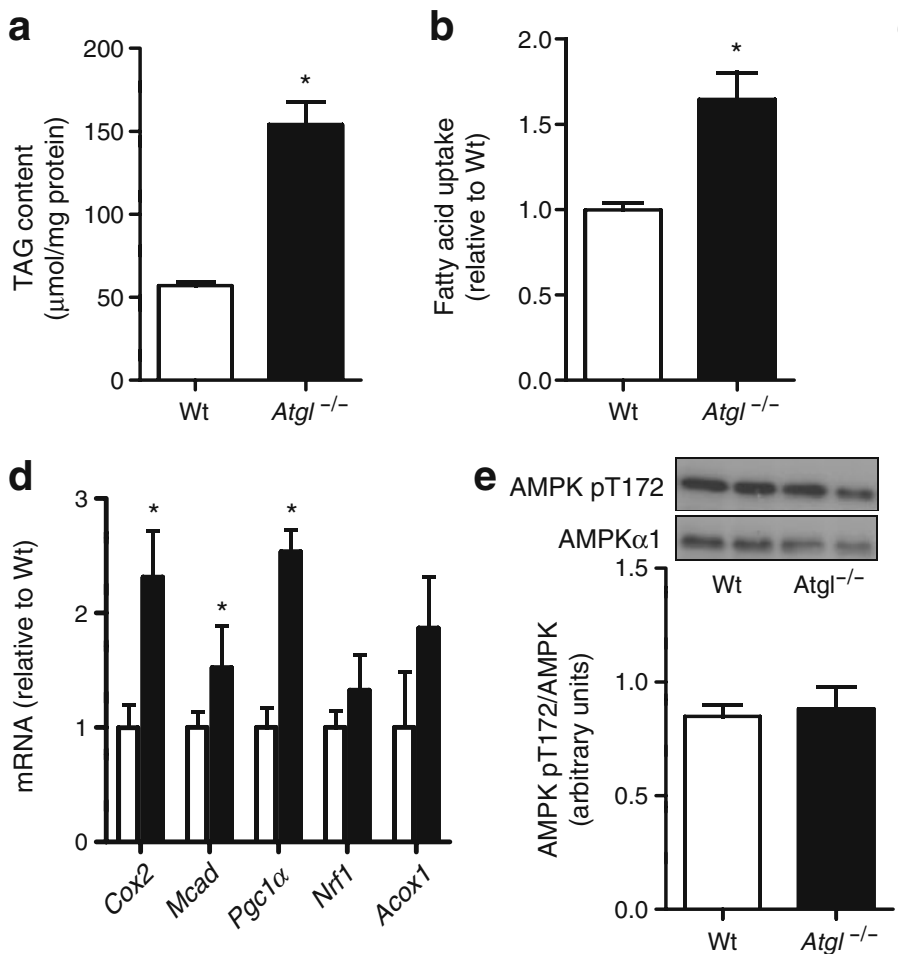

h

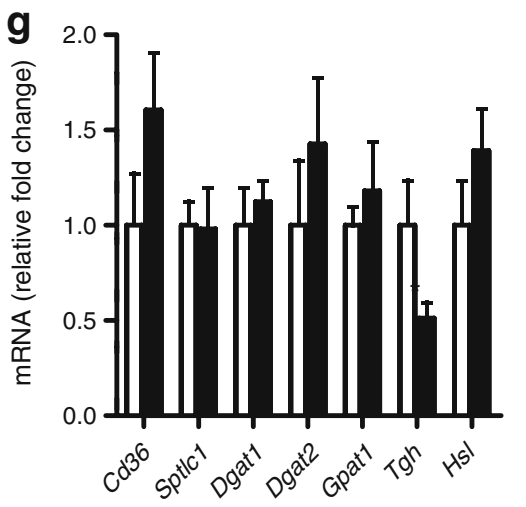

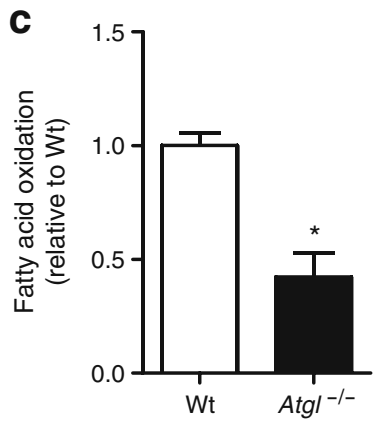

f

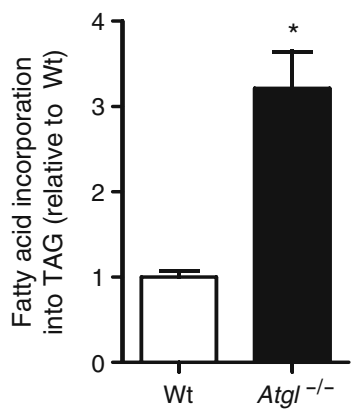

i
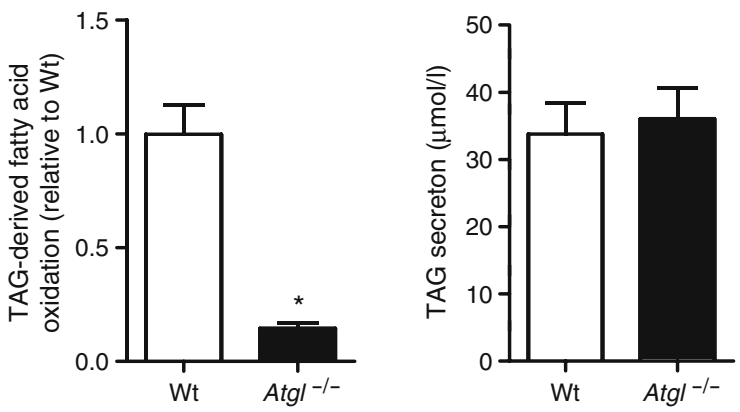

larger in Ad-GFP compared with Ad-ATGL mice and quantitative biochemical analysis of liver revealed a marked reduction in the intracellular TAG content of Ad-ATGL mice (Fig. 4d). Mediators of fatty acid storage, including sterol regulatory element-binding protein 1, stearoylcoenzyme A desaturase 1 and fatty acid synthase were concomitantly decreased with Ad-ATGL (Fig. 4e). The lipids diacylglycerol $(32 \%)$ and ceramide $(34 \%)$ were reduced with Ad-ATGL (Fig. 4f, g). Interestingly, fasting plasma NEFA was increased in Ad-ATGL (Table 1) despite no change in basal and $\beta$-adrenergic lipolytic capacity as assessed in adipose tissue explants ex vivo (data not shown). While an increase in plasma NEFA positively correlates with TAG accumulation in ectopic tissues [29], we detected no increase in intracellular TAG deposition in skeletal muscle or heart with Ad-ATGL (Table 1). TAG secretion rate and plasma TAGs were not increased in AdATGL compared with Ad-GFP (Table 1). Plasma $\beta$ - hydroxybutyrate was elevated in Ad-ATGL mice (Table 1), suggesting enhanced $\beta$-oxidation in the liver.

Hepatic ATGL overproduction mildly increases hepatic insulin sensitivity Because Ad-ATGL induced a marked reduction in hepatic steatosis, we postulated that insulin sensitivity may be improved in these mice. Fasting blood glucose tended to be lower in Ad-ATGL $(12 \%, p=0.08)$ and plasma insulin was not different between groups (decreased by $33 \%$ in Ad-ATGL, $p=0.17$, Table 1 ) 2 weeks after adenovirus administration. Whole-body insulin sensitivity was mildly improved with Ad-ATGL (Fig. 5a), but these changes were not accompanied by enhanced skeletal muscle insulin responsiveness assessed ex vivo (Fig. 5b). The mild changes in whole-body glucose metabolism were accompanied by the suppression of hepatic $P c g-1 \alpha$ (also known as Ppargcla; Fig. 5c), which stimulates the production of gluconeogenic enzymes [31]. Consistent with 
Fig. 3 Atgl ablation does not appreciably influence insulin action in primary hepatocytes. Hepatocytes isolated from $\mathrm{Wt}$ and $\operatorname{Atg}^{/-}$mice were cultured for 2-3 days prior to assays. (a) Akt phosphorylation at S473 and (b) T308 under basal conditions or after acute insulin stimulation ( $n=3-4$ per condition). In separate experiments, hepatocytes were incubated with insulin (0-10 nmol/l) for $4 \mathrm{~h}$ and Pepck (c) and G6Pase (d) mRNA was determined by qRT-PCR ( $n=4-12$ per condition). e Hepatic glucose output was assessed in Wt (white bars) and $A \operatorname{tg} \Gamma^{1-}$ hepatocytes (black bars) under basal and insulin stimulated conditions ( $n=6$ per group). f Hepatic glucose output reported as \% suppression by insulin. Wt (white bars) and $A \operatorname{tg} \Gamma^{\prime-}$

(black bars) ( $n=6$ per group)
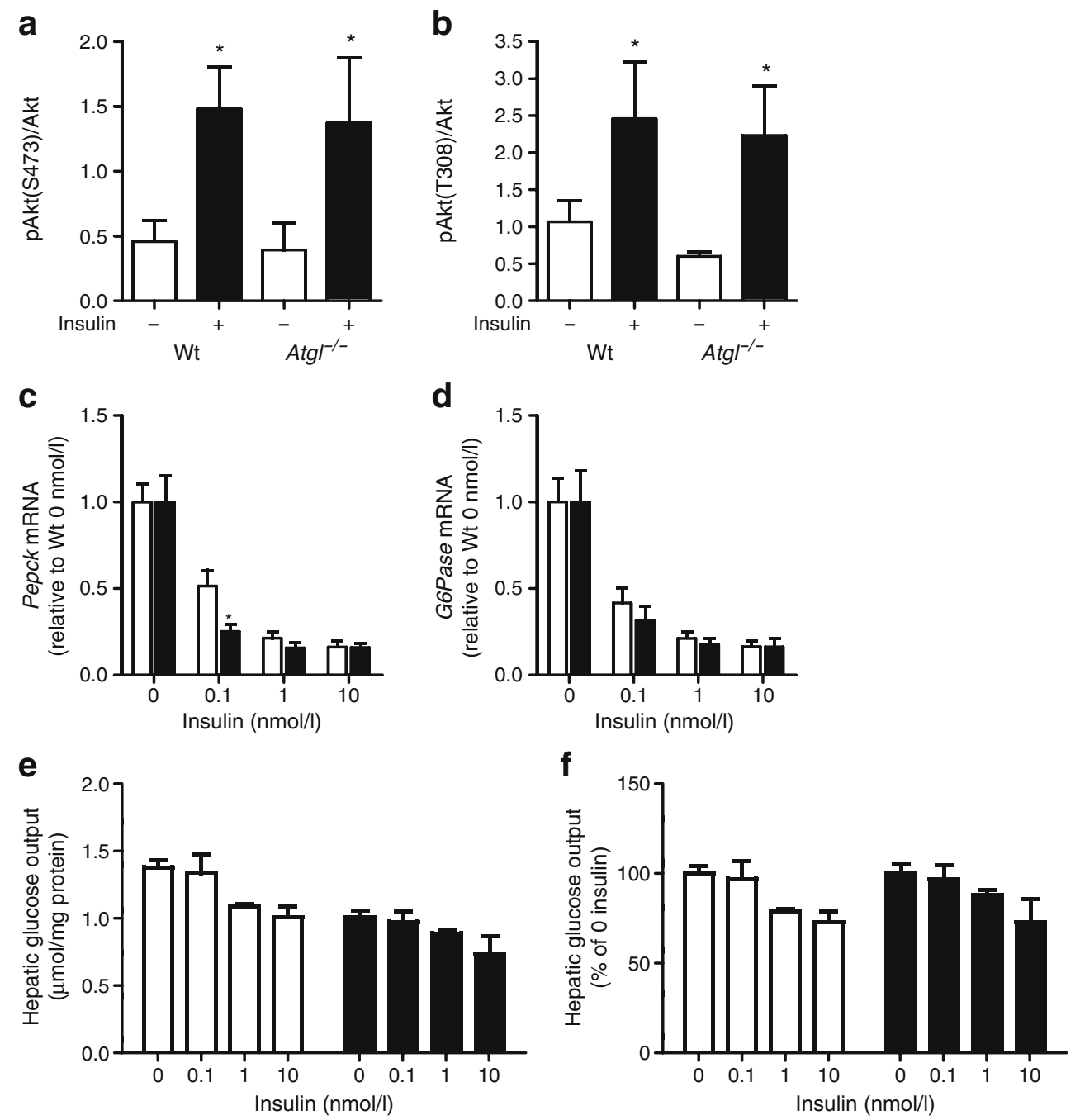

this effect, Pepck mRNA was suppressed in livers of AdATGL mice (Fig. 5c). There was no difference in G6Pase mRNA. We tested the role of Ad-ATGL in modulating gluconeogenesis in vivo by examining glucose levels after the i.p. administration of pyruvate. The glucose area under the curve tended $(p=0.09)$ to be reduced in Ad-ATGL after a high pyruvate load $(2 \mathrm{mg} / \mathrm{kg})$, suggestive of reduced gluconeogenesis (Fig. 5d). We repeated this experiment with a lower pyruvate dose $(0.35 \mathrm{mg} / \mathrm{kg})$ to determine the sensitivity of the gluconeogenic response and detected a reduced glucose excursion with Ad-ATGL (Fig. 5e). Also, the phosphorylation of Akt and its downstream target forkhead box $\mathrm{O}$ (FOXO)1 (Fig. 5f-h) were increased in Ad-ATGL mice vs Ad-GFP. Collectively, these data demonstrate that enhanced hepatic insulin sensitivity in Ad-ATGL mice contributes to a subtle enhancement of whole-body insulin action.

Hepatic ATGL overproduction has no effect on inflammatory signalling Hepatic steatosis can be accompanied by inflam- mation that is likely to contribute to insulin resistance [32]. We examined several markers of intracellular inflammation in the livers of mice. The mRNA expression of the proinflammatory cytokine TNF- $\alpha$ was reduced $(p=0.06)$ in Ad-ATGL vs Ad-GFP (ESM Fig. 1a), whereas $I l 6$ and suppressor of cytokine signalling 3 expression were not different between groups (ESM Fig. 1a). There was no difference in CD68 protein content, suggesting no effect of Ad-ATGL on Kupffer cell numbers (ESM Fig. 1a).

Increased TNF $\alpha$ [33] and lipid accumulation leads to activation of serine/threonine kinases that can decrease insulin signalling [32]. Despite the reduced hepatic TNF $\alpha$ content and lipid accumulation in Ad-ATGL, the phosphorylation levels of c-Jun N-terminal kinase (JNK; ESM Fig. 1b) and activation of the nuclear factor-kappa $\mathrm{B}(\mathrm{NF} \kappa \mathrm{B})$ pathway, as indicated by nuclear factor of kappa $\mathrm{B}$ inhibitor alpha $(\mathrm{I} K \mathrm{~B} \alpha)$ degradation (ESM Fig. 1c), were not different. Protein kinase $\mathrm{C}(\mathrm{PKC}) \varepsilon$ is activated by diacylglycerol and has been implicated in the development of hepatic insulin resistance [34]. There was no difference in the membrane to 
Fig. 4 Hepatic Atgl expression reduces lipid deposition in livers of obese mice. Adenovirus containing GFP (white bars) or ATGL (black bars) was intravenously administered to mice and tissues were collected after 14 days. a $A t g l$ mRNA was determined by qRT-PCR $(n=10-13)$. b Representative immunoblot showing ATGL $(54 \mathrm{kDa})$ and ATGL-GFP (76 kDa). c Sectioned livers stained with Oil Red O demonstrate marked steatosis in Ad-GFP mice and a reduction in staining with Ad-ATGL. d Liver triacylglycerol was assessed biochemically $(n=8-9$ per group). e Srebf1, Scd1 and Fas mRNA were assessed by qRT-PCR ( $n=3-8$ per group). ${ }^{\dagger} p=0.06$. Liver diacylglycerol (DAG) (f) and ceramide (g) were determined in Ad-GFP and Ad-ATGL mice $(n=10-13$ per group). ${ }^{*}$ Different from Ad-GFP, $p<0.05$ a

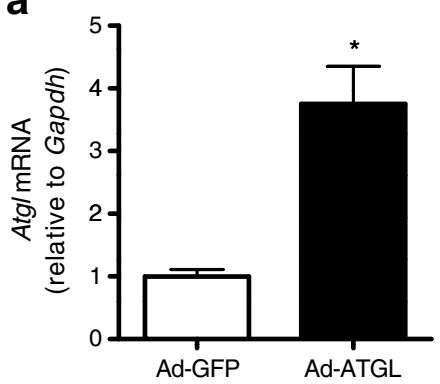

C

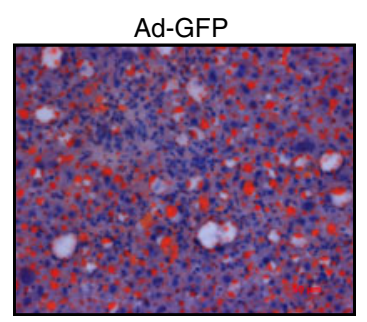

e

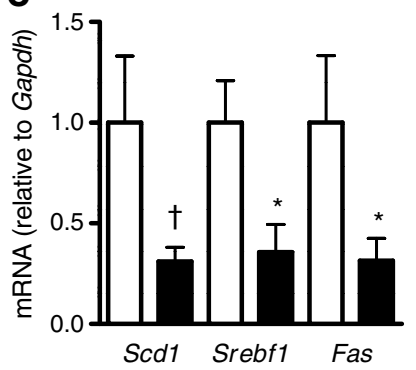

b
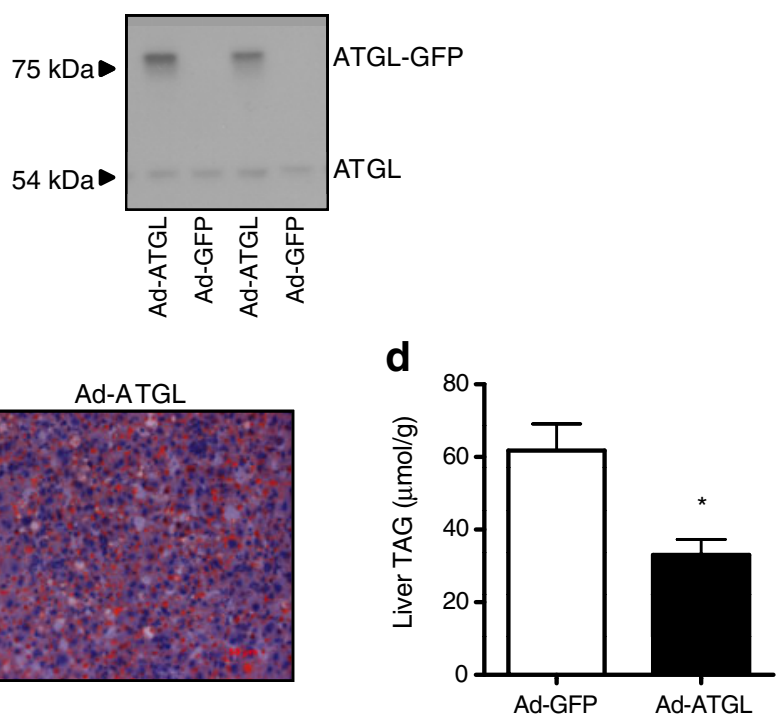

f
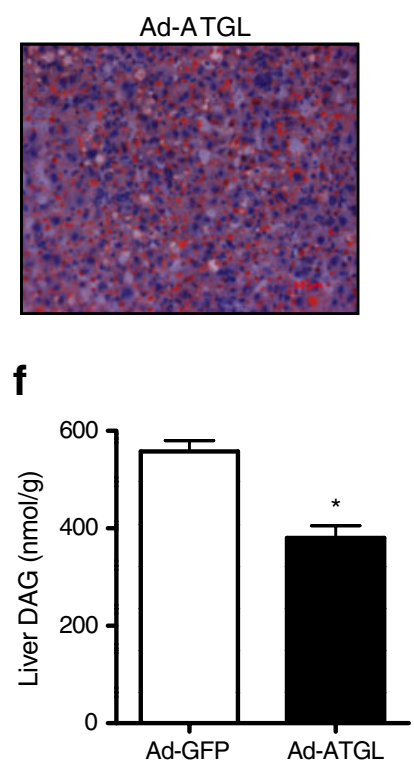

cytosolic ratio (marker of PKC activity) of $\mathrm{PKC} \varepsilon$ (ESM Fig. 1d) or PKC $\theta$ (data not shown). Thus, the improvement in hepatic insulin action with ATGL overproduction was not a consequence of diminished inflammatory pathway activation in the liver.

\section{Discussion}

ATGL is a critical regulator of triacylglycerol hydrolysis in most metabolic tissues [19] and truncated ATGL induces clinical symptoms including hepatic TAG accumulation and hepatomegaly [21]. The goal of this study was to directly assess the role of ATGL in hepatic lipid metabolism and insulin sensitivity. We show that ablation of Atgl in hepatocytes increased TAG storage by reducing the capacity to mobilise TAG-derived fatty acids for oxidation, without affecting TAG secretion. Surprisingly, insulin sensitivity was not impaired in Atgl-null hepatocytes despite the marked lipid accumulation. Hepatic overproduction of ATGL in obese mice reduced hepatic lipid content without influencing macrophage infiltration or local g

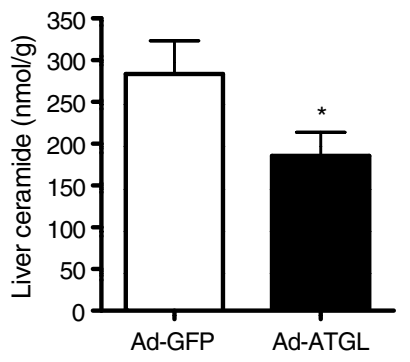

inflammation. ATGL overproduction led to subtle improvements in hepatic insulin responsiveness that did not translate into notable changes in whole-body insulin sensitivity, fasting blood glucose or insulin levels. These findings provide novel insights into the role of ATGL in hepatic TAG metabolism and provide specific evidence that ATGL is not a major mediator of hepatic insulin sensitivity.

Steatosis is characterised by TAG deposition and a major contributor is enhanced fatty acid availability from dysregulated adipose tissue lipolysis [35]. The susceptibility of the liver to fatty acid fluxes is hardly surprising, as visceral fat directly drains fatty acids into the liver via the portal vein. While this is less studied, a growing body of evidence indicates that the modification of TAG synthesis or degradation processes contributes to steatosis development [11, 22, 36-38].

Unlike a previous study [22], we closely examined ATGL loss of function in isolated hepatocytes to avoid the potential for confounding regulation introduced by neural, hormonal and inflammatory factors. Atgl deletion induced several alterations in fatty acid handling that resulted in TAG accumulation. An important implication of our studies is that decreased fatty acid oxidation contributes to steatosis in 
Table 1 Metabolic characteristics of obese C57Bl/6 mice treated with Ad-GFP and Ad-ATGL

\begin{tabular}{lcc}
\hline Characteristic & Ad-GFP & Ad-ATGL \\
\hline Body mass (g) & $39.1 \pm 1.3$ & $39.6 \pm 1.0$ \\
Lean mass (g) & $27.3 \pm 0.5$ & $26.5 \pm 0.5$ \\
Fat mass (g) & $13.9 \pm 1.0$ & $13.0 \pm 1.2$ \\
Liver mass (g) & $1.51 \pm 0.09$ & $1.49 \pm 0.11$ \\
Food intake (g/day) & $2.3 \pm 0.2$ & $2.2 \pm 0.2$ \\
Serum ALT (U/l) & $168 \pm 24$ & $230 \pm 22$ \\
Plasma glucose (mmol/l) & $8.98 \pm 0.44$ & $7.95 \pm 0.36$ \\
Plasma insulin (pmol/l) & $117 \pm 22$ & $78 \pm 15$ \\
Plasma NEFA (mmol/l) & $0.64 \pm 0.07$ & $0.84 \pm 0.05^{*}$ \\
Plasma TAG (mmol/l) & $0.79 \pm 0.12$ & $0.91 \pm 0.12$ \\
TAG secretion rate $\left(\mathrm{mmoll}{ }^{-1} \mathrm{~h}^{-1}\right.$ ) & $0.68 \pm 0.37$ & $0.31 \pm 0.10$ \\
Plasma $\beta$-hydroxybutyrate $(\mu \mathrm{mol} / \mathrm{l})$ & $171 \pm 30$ & $420 \pm 109^{*}$ \\
Skeletal muscle TAG $(\mu \mathrm{mol} / \mathrm{g})$ & $37.3 \pm 8.2$ & $31.3 \pm 9.3$ \\
Heart TAG $(\mu \mathrm{mol} / \mathrm{g})$ & $16.8 \pm 1.4$ & $15.7 \pm 1.3$ \\
\hline
\end{tabular}

All measures were obtained in mice fasted for $4 \mathrm{~h}$. Body mass and fat mass were determined on day 14 . Food intake was measured daily from day 10 to day 14 .

Values are mean \pm SEM, $n=4-13$

${ }^{*} p<0.05$ vs Ad-GFP

$A \operatorname{tg} \Gamma^{/-}$hepatocytes. Gene expression of proteins involved in mitochondrial fatty acid oxidation was actually increased in $A \operatorname{tg} \Gamma^{-1}$ hepatocytes, suggesting that the defect in hepatic fatty acid oxidation was independent of defective protein production. Instead, the oxidation of fatty acids derived from intraand extracellular sources was reduced by $58 \%$ and $85 \%$, respectively, supporting the premise that most of the fatty acids entering the liver are first incorporated into TAG prior to oxidation. Thus, the critical and novel observation is that an inability to mobilise TAG-derived fatty acids contributes to the reduction in oxidation and steatosis. In support of this idea, ATGL overproduction in rat hepatoma cells decreased cellular TAG and increased fatty acid oxidation [22]. Also, downregulation of the ATGL co-activator CGI-58 reduced fatty acid oxidation whereas CGI-58-mediated TAG hydrolysis was coupled to enhanced fatty acid oxidation in HepG2 cells [23]. The concept that plasma-derived fatty acids first enter the TAG pool within intracellular lipid droplets before their eventual oxidation was suggested by Gibbons et al. [14] and experimentally shown in skeletal muscle [39]. A second implication from these studies is that hepatocytes continue to take up fatty acids despite marked steatosis. The absence of a powerful negative feedback loop may reflect the requirement of the liver to utilise fatty acid substrate for gluconeogenesis, which occurs during starvation with concurrent hepatic lipid accumulation [40], or may reflect the requirement of the liver to clear and package excess plasma NEFA that are invariably toxic to most tissues [2].
A previous study reported reduced TAG content in obese mice after massive (200-fold) ATGL and HSL adenovirus administration [22]. We also found that modest ATGL overproduction reduced TAG content in the livers of obese mice. The decrease in TAG content was most likely due to increased TAG lipolysis and oxidation of the liberated fatty acids. Consistent with increased rates of fatty acid oxidation in livers of Ad-ATGL mice, we detected elevated fasting plasma $\beta$-hydroxybutyrate. Our results showing no increase in circulating TAG or lipid deposition in other ectopic tissues (skeletal muscle, heart, adipose tissue) with AdATGL are consistent with the notion that TAG export is unaffected by hepatic ATGL overproduction in vivo (Table 1) [22] or hepatic ATGL ablation in vitro (Fig. 2i). We were unable to complete these measures in $\mathrm{Atg}^{-1}$ mice because of unacceptable mortality. Collectively, these data indicate that ATGL directs TAG-derived fatty acids towards oxidation. In contrast, the other major TAG lipase, TGH, preferentially directs fatty acids from TAG towards VLDL production [41]. Thus, while ATGL and TGH both possess TAG lipase activity, they appear to channel the liberated fatty acids towards oxidation and VLDL packaging, respectively.

The fatty liver of insulin-resistant mice is attributed in part to increased production and activity of sterol regulatory element binding protein (SREBP)-1c and subsequent upregulation of lipogenic gene expression [42]. Our findings of reduced SREBP-1c, and its downstream targets FAS and SCD1, and a tendency for reduced fasting plasma insulin support the possibility that TAG synthesis rates were reduced with Ad-ATGL. A major issue of clinical relevance is whether providing metabolic relief by directing lipids away from the liver is a sound approach for the treatment of steatosis/fatty liver and insulin resistance, or whether such an approach would give rise to compensatory lipid storage in other tissues and the induction of secondary complications [43]. A critical finding from this study is that hepatic ATGL overproduction does not result in the redistribution/ partitioning of lipids into other tissues, supporting the practicality of enhancing ATGL production by pharmacological manipulation for treating fatty liver.

The role of ATGL in hepatic insulin action is unresolved, with reports of increased [19] and decreased [30] insulin sensitivity in whole-body $\mathrm{Atg}^{-1}$ mice. Here, we directly examined the effect of ATGL on hepatic insulin sensitivity. Studies in isolated hepatocytes demonstrated normal insulin sensitivity in $\mathrm{Atgl}^{--}$hepatocytes despite marked TAG accumulation. This is consistent with recent studies demonstrating a mismatch between steatosis and insulin sensitivity $[10-12,22]$ and suggests that lipid accumulation per se is not sufficient to induce insulin resistance. In contrast, increasing liver ATGL mildly improved hepatic insulin action in obese insulin-resistant mice. These functional outcomes were mechanistically underpinned by suppression 
Fig. 5 Ad-ATGL improves insulin sensitivity in mice. a Mice treated with Ad-GFP or Ad-ATGL were injected i.p. with $1 \mathrm{U} / \mathrm{kg}$ insulin and blood glucose was monitored for $120 \mathrm{~min}$. White circles, AdGFP; black circles, Ad-ATGL ( $n=11-13$ per group). ${ }^{*} p<0.05$ for main effect for treatment. b Glucose uptake into soleus muscle was determined ex vivo under basal or insulin stimulated (10 nmol/l) conditions

( $n=6-8$ per condition); ${ }^{*} p<0.05$ vs basal within the same treatment. c Pepck, G6Pase and $P g c-1 \alpha$ (also known as Ppargcla) mRNA in the liver of mice after a 4-6 h fast. (d, e) Mice were injected i.p. with a high $(2 \mathrm{~g} / \mathrm{kg})(\mathbf{d})$ or low $(0.35 \mathrm{~g} / \mathrm{kg})$ (e) pyruvate load, and blood glucose was assessed for 60-90 min. White circles, Ad-GFP; black circles, Ad-ATGL ( $n=3-6$ per group). f-h Mice were anaesthetised with isoflurane and injected $i . v$. with $5 \mathrm{U} / \mathrm{kg}$ dose insulin. Livers were freeze clamped after $10 \mathrm{~min}$ and insulin sensitivity was assessed by measuring (f) pAkt (S473) and (g) pFOXO1 (S256). ${ }^{*} p<0.05,{ }^{\dagger} p=0.058$ vs Ad-GFP. Representative blots are shown in (h) $(n=7-8$ per group)
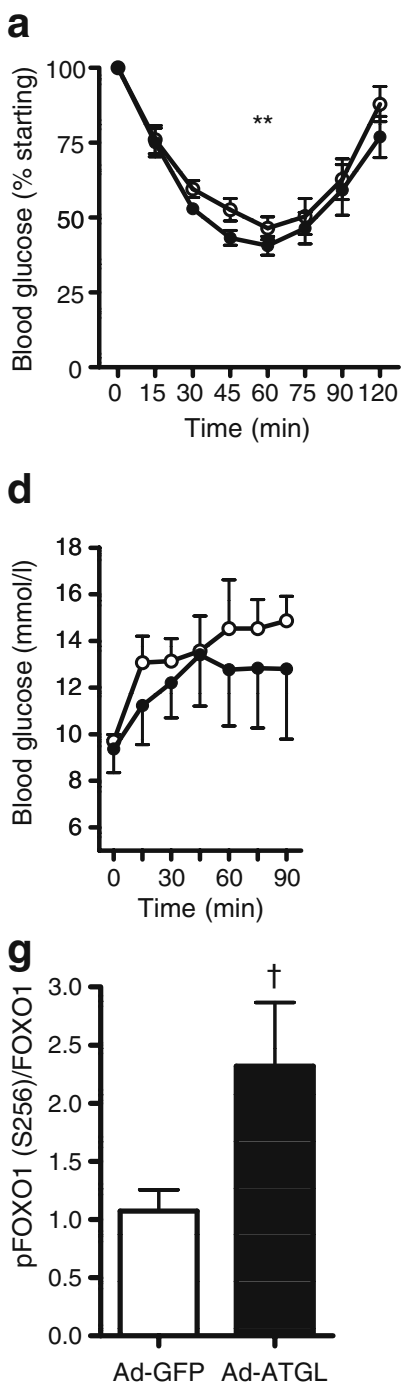

b

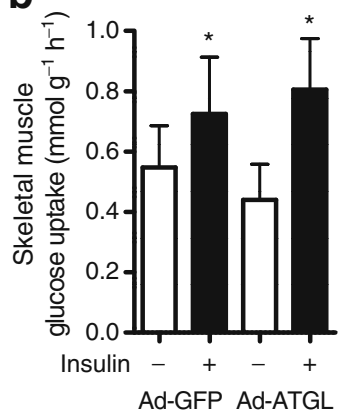

e

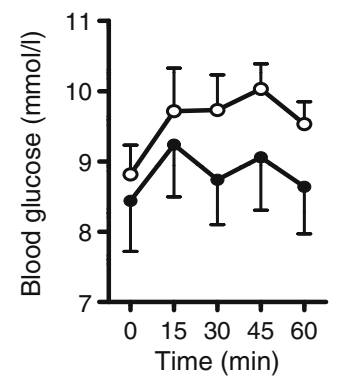

h

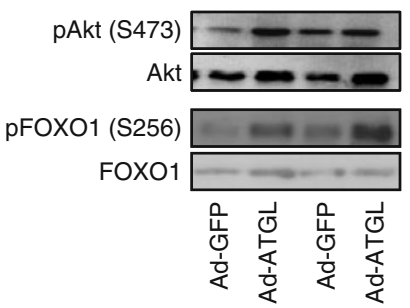

C

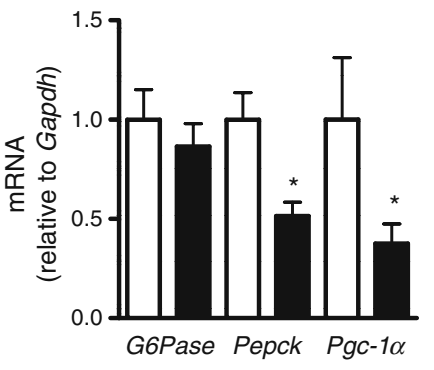

f

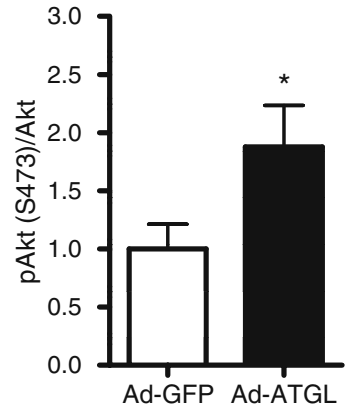

of PGC- $1 \alpha$ and phosphoenolpyruvate kinase (PEPCK) in fasting mice, improved insulin signal transduction and suppression of gluconeogenesis following pyruvate exposure. These changes were not sufficient to reduce fasting glycaemia and insulinaemia.

Hepatic steatosis is closely linked to insulin resistance; however, TAGs are unlikely to directly cause insulin resistance because they are stored within lipid droplets. Also, TAG accumulates during fasting when insulin sensitivity is not impaired, refuting the likelihood of a causal negative relationship. Other metabolically active lipid intermediates, such as diacylglycerol and ceramide, are mechanistically linked to defective insulin signal transduction. There is considerable associative evidence to support a role for diacylglycerol in mediating hepatic insulin resistance, with the demonstration of concurrent reductions in diacylglycerol, $\mathrm{PKC} \varepsilon$ activity and improved insulin receptor substrate signalling following various experimental manipulations $[34,36,37]$. While we showed that ATGL overproduction reduced liver diacylglycerol by $\sim 30 \%$, PKC $\varepsilon$ membrane translocation was unaffected. Other serine/threonine kinases such as JNK and inhibitor of kappa kinase are implicated in the development of insulin resistance [44, 45], yet neither kinase was attenuated with ATGL overproduction. Ceramide inhibits insulin signalling at Akt by dephosphorylation mediated by protein phosphatase 2A [46] and by preventing the translocation of Akt [47]. Pharmacological and genetic interference of ceramide biosynthesis is associated with enhanced insulin sensitivity [48]. Our studies show that ATGL overproduction reduced ceramide content and increased Akt phosphorylation in the liver of obese mice, supporting the concept that ATGL influences distal hepatic insulin signalling [30]. Interestingly, ceramide was not increased in $\mathrm{Atgl} \Gamma^{/-}$ hepatocytes, which may explain the maintenance of insulin sensitivity in these cells.

In summary, this study demonstrates that hepatic ATGL production is reduced in several models of rodent obesity and that ATGL ablation leads to pronounced steatosis that 
is characterised by an impairment in fatty acid oxidation. Increasing ATGL production in the liver reduces hepatic steatosis and mildly enhances liver insulin sensitivity. These effects are not sufficient to improve fasting glycaemia or insulinaemia in rodent obesity, suggesting that ATGL represents a pharmacological therapeutic target for fatty liver disease but not type 2 diabetes.

Acknowledgements We thank G. Haemmerle and R. Zechner (University of Graz) for the $A \operatorname{tg} \Gamma^{-1}$ mice and C. Schmitz-Peiffer for the $\mathrm{PKC} \varepsilon$ antibody. These studies were supported by research grants from the National Health and Medical Research Council (NHMRC) of Australia and a Monash Fellowship. S. M. Turpin was supported by a NHMRC Dora Lush Biomedical Postgraduate Scholarship. A. J. Hoy is supported by a Biomedical Australian training fellowship and M. J. Watt a Senior Research Fellowship from the NHMRC.

Duality of interest The authors declare that there is no duality of interest associated with this manuscript.

\section{References}

1. Friedman J (2002) Fat in all the wrong places. Nature 415:268-269

2. Unger RH (2003) Minireview: weapons of lean body mass destruction: the role of ectopic lipids in the metabolic syndrome. Endocrinology 144:5159-5165

3. Almeda-Valdes P, Cuevas-Ramos D, Aguilar-Salinas CA (2009) Metabolic syndrome and non-alcoholic fatty liver disease. Ann Hepatol 8(Suppl 1):S18-S24

4. Biddinger SB, Kahn CR (2006) From mice to men: insights into the insulin resistance syndromes. Annu Rev Physiol 68:123-158

5. Bock G, Chittilapilly E, Basu R et al (2007) Contribution of hepatic and extrahepatic insulin resistance to the pathogenesis of impaired fasting glucose: role of increased rates of gluconeogenesis. Diabetes 56:1703-1711

6. Gastaldelli A, Baldi S, Pettiti M et al (2000) Influence of obesity and type 2 diabetes on gluconeogenesis and glucose output in humans: a quantitative study. Diabetes 49:1367-1373

7. Halaas JL, Gajiwala KS, Maffei M et al (1995) Weight-reducing effects of the plasma protein encoded by the obese gene. Science 269:543-546

8. Shimomura I, Matsuda M, Hammer RE, Bashmakov Y, Brown MS, Goldstein JL (2000) Decreased IRS-2 and increased SREBP1c lead to mixed insulin resistance and sensitivity in livers of lipodystrophic and $o b / o b$ mice. Mol Cell 6:77-86

9. Oakes ND, Cooney GJ, Camilleri S, Chisholm DJ, Kraegen EW (1997) Mechanisms of liver and muscle insulin resistance induced by chronic high-fat feeding. Diabetes 46:1768-1774

10. Kantartzis K, Machicao F, Machann J et al (2009) The DGAT2 gene is a candidate for the dissociation between fatty liver and insulin resistance in humans. Clin Sci (Lond) 116:531-537

11. Monetti M, Levin MC, Watt MJ et al (2007) Dissociation of hepatic steatosis and insulin resistance in mice overexpressing DGAT in the liver. Cell Metab 6:69-78

12. Solinas G, Vilcu C, Neels JG et al (2007) JNK1 in hematopoietically derived cells contributes to diet-induced inflammation and insulin resistance without affecting obesity. Cell Metab 6:386-397

13. Nagle CA, Klett EL, Coleman RA (2009) Hepatic triacylglycerol accumulation and insulin resistance. J Lipid Res 50(Suppl):S74-S79

14. Gibbons GF, Wiggins D, Brown AM, Hebbachi AM (2004) Synthesis and function of hepatic very-low-density lipoprotein. Biochem Soc Trans 32:59-64
15. Francone OL, Kalopissis AD, Griffaton G (1989) Contribution of cytoplasmic storage triacylglycerol to VLDL-triacylglycerol in isolated rat hepatocytes. Biochim Biophys Acta 1002:28-36

16. Odegaard JI, Ricardo-Gonzalez RR, Red Eagle A et al (2008) Alternative M2 activation of Kupffer cells by PPAR $\delta$ ameliorates obesity-induced insulin resistance. Cell Metab 7:496-507

17. Koonen DP, Jacobs RL, Febbraio M et al (2007) Increased hepatic CD36 expression contributes to dyslipidemia associated with dietinduced obesity. Diabetes 56:2863-2871

18. Zimmermann R, Strauss JG, Haemmerle G et al (2004) Fat mobilization in adipose tissue is promoted by adipose triglyceride lipase. Science 306:1383-1386

19. Haemmerle G, Lass A, Zimmermann R et al (2006) Defective lipolysis and altered energy metabolism in mice lacking adipose triglyceride lipase. Science 312:734-737

20. Lass A, Zimmermann R, Haemmerle G et al (2006) Adipose triglyceride lipase-mediated lipolysis of cellular fat stores is activated by CGI-58 and defective in Chanarin-Dorfman Syndrome. Cell Metab 3:309-319

21. Kobayashi K, Inoguchi T, Maeda Y et al (2008) The lack of the Cterminal domain of adipose triglyceride lipase causes neutral lipid storage disease through impaired interactions with lipid droplets. J Clin Endocrinol Metab 93:2877-2884

22. Reid BN, Ables GP, Otlivanchik OA et al (2008) Hepatic overexpression of hormone-sensitive lipase and adipose triglyceride lipase promotes fatty acid oxidation, stimulates direct release of free fatty acids, and ameliorates steatosis. J Biol Chem 283: 13087-13099

23. Brown JM, Chung S, Das A, Shelness GS, Rudel LL, Yu L (2007) CGI-58 facilitates the mobilization of cytoplasmic triglyceride for lipoprotein secretion in hepatoma cells. J Lipid Res 48:2295-2305

24. Caviglia JM, Sparks JD, Toraskar N et al (2009) ABHD5/CGI-58 facilitates the assembly and secretion of apolipoprotein B lipoproteins by McA RH7777 rat hepatoma cells. Biochim Biophys Acta 1791:198-205

25. Kato M, Higuchi N, Enjoji M (2008) Reduced hepatic expression of adipose tissue triglyceride lipase and CGI-58 may contribute to the development of non-alcoholic fatty liver disease in patients with insulin resistance. Scand J Gastroenterol 43:1018-1019

26. Schoenborn V, Heid IM, Vollmert C et al (2006) The ATGL gene is associated with free fatty acids, triglycerides, and type 2 diabetes. Diabetes 55:1270-1275

27. Scott JW, van Denderen BJ, Jorgensen SB et al (2008) Thienopyridone drugs are selective activators of AMP-activated protein kinase $\beta 1$-containing complexes. Chem Biol 15:12201230

28. Watt MJ, Steinberg GR, Chen ZP, Kemp BE, Febbraio MA (2006) Fatty acids stimulate AMP-activated protein kinase and enhance fatty acid oxidation in L6 myotubes. J Physiol 574:139-147

29. Watt MJ, Hevener A, Lancaster GI, Febbraio MA (2006) Ciliary neurotrophic factor prevents acute lipid-induced insulin resistance by attenuating ceramide accumulation and phosphorylation of cJun N-terminal kinase in peripheral tissues. Endocrinology 147: 2077-2085

30. Kienesberger PC, Lee D, Pulinilkunnil T et al (2009) Adipose triglyceride lipase (ATGL) deficiency causes tissue-specific changes in insulin signaling. J Biol Chem 284:30218-30229

31. Yoon JC, Puigserver P, Chen G et al (2001) Control of hepatic gluconeogenesis through the transcriptional coactivator PGC-1. Nature 413:131-138

32. Wellen KE, Hotamisligil GS (2005) Inflammation, stress, and diabetes. J Clin Invest 115:1111-1119

33. Fujishiro M, Gotoh Y, Katagiri H et al (2003) Three mitogenactivated protein kinases inhibit insulin signaling by different mechanisms in 3T3-L1 adipocytes. Mol Endocrinol 17:487-497 
34. Samuel VT, Liu ZX, Wang A et al (2007) Inhibition of protein kinase Cepsilon prevents hepatic insulin resistance in nonalcoholic fatty liver disease. J Clin Invest 117:739-745

35. Donnelly KL, Smith CI, Schwarzenberg SJ, Jessurun J, Boldt MD, Parks EJ (2005) Sources of fatty acids stored in liver and secreted via lipoproteins in patients with nonalcoholic fatty liver disease. J Clin Invest 115:1343-1351

36. Choi CS, Savage DB, Kulkarni A et al (2007) Suppression of diacylglycerol acyltransferase-2 (DGAT2), but not DGAT1, with antisense oligonucleotides reverses diet-induced hepatic steatosis and insulin resistance. J Biol Chem 282:22678-22688

37. Neschen S, Morino K, Hammond LE et al (2005) Prevention of hepatic steatosis and hepatic insulin resistance in mitochondrial acyl-CoA:glycerol-sn-3-phosphate acyltransferase 1 knockout mice. Cell Metab 2:55-65

38. Yu XX, Murray SF, Pandey SK et al (2005) Antisense oligonucleotide reduction of DGAT2 expression improves hepatic steatosis and hyperlipidemia in obese mice. Hepatology 42:362-371

39. Kanaley JA, Shadid S, Sheehan MT, Guo Z, Jensen MD (2009) Relationship between plasma FFA, intramyocellular triglycerides and long-chain acylcarnitines in resting humans. J Physiol 587:5939-5950

40. Moller L, Stodkilde-Jorgensen H, Jensen FT, Jorgensen JO (2008) Fasting in healthy subjects is associated with intrahepatic accumulation of lipids as assessed by ${ }^{1} \mathrm{H}$-magnetic resonance spectroscopy. Clin Sci (Lond) 114:547-552
41. Lehner R, Vance DE (1999) Cloning and expression of a cDNA encoding a hepatic microsomal lipase that mobilizes stored triacylglycerol. Biochem J 343(Pt 1):1-10

42. Horton JD, Goldstein JL, Brown MS (2002) SREBPs: activators of the complete program of cholesterol and fatty acid synthesis in the liver. J Clin Invest 109:1125-1131

43. Hoy AJ, Watt MJ (2009) Lipid partitioning and insulin sensitivity: lessons learnt from genetic models. APJOE 1:35-47

44. Hirosumi J, Tuncman G, Chang L et al (2002) A central role for JNK in obesity and insulin resistance. Nature 420:333-336

45. Yuan M, Konstantopoulos N, Lee J et al (2001) Reversal of obesity- and diet-induced insulin resistance with salicylates or targeted disruption of Ikk $\beta$. Science 293:1673-1677

46. Schmitz-Peiffer C, Craig DL, Biden TJ (1999) Ceramide generation is sufficient to account for the inhibition of the insulin-stimulated pkb pathway in $\mathrm{C}_{2} \mathrm{C}_{12}$ skeletal muscle cells pretreated with palmitate. J Biol Chem 274:24202-24210

47. Cazzolli R, Carpenter L, Biden TJ, Schmitz-Peiffer C (2001) A role for protein phosphatase 2a-like activity, but not atypical protein kinase $\mathrm{C} \zeta$, in the inhibition of protein kinase b/akt and glycogen synthesis by palmitate. Diabetes 50:2210-2218

48. Holland WL, Brozinick JT, Wang LP et al (2007) Inhibition of ceramide synthesis ameliorates glucocorticoid-, saturatedfat-, and obesity-induced insulin resistance. Cell Metab 5: $167-179$ 